\title{
Axial Vibration of Embedded Nanorods Under Transverse Magnetic Field Effects via Nonlocal Elastic Continuum Theory
}

\author{
T. Murmu ${ }^{1}, *$, S. Adhikari², and M. A. McCarthy ${ }^{1}$ \\ ${ }^{1}$ Department of Mechanical, Aeronautical and Biomedical Engineering, Irish Centre for Composites Research, \\ Materials and Surface Science Institute, University of Limerick, Ireland \\ ${ }^{2}$ College of Engineering, Swansea University, Singleton Park, Swansea SA2 8PP, Wales, UK
}

\begin{abstract}
In this paper, the influence of a transverse magnetic field on the axial vibration of nanorods such as carbon nanotubes is theoretically modelled using nonlocal elasticity approach. Nonlocal elasticity handles the small-scale effects of vibrating nanorod. Nonlocal rod theory is utilised and detailed analytical solutions are obtained. The nanorod is assumed sensitive to magnetic field. Governing equations for nonlocal axial vibration of the nanorod under a transverse magnetic field are derived considering the Lorentz magnetic force obtained from Maxwell's relation. Nonlocal rod embedded in an elastic medium is also considered. Clamped-clamped and clamped-free boundary conditions are considered. A simple analytical expression for the natural frequencies is proposed. Results from the analytical model developed show that the transverse magnetic field exerted on the nanorod theoretically dampens the nonlocal effect of atom-atom interactions by increasing the natural frequencies. The variation of frequency with the increase of axial stiffness of elastic medium for an embedded nanorod in a magnetic field is more nonlinear with nonlocal effect than without nonlocal effects. This study provides the necessary physical insights for experimental studies on the dynamics of magnetically sensitive nanorods.
\end{abstract}

Keywords: Nonlocal Elasticity, Magnetic Field, Axial Vibration, Nanorods.

\section{INTRODUCTION}

With the advent of nanotechnology, studies on the properties nanostructures are gaining popularity. ${ }^{1,2}$ Recently a new area of research has evolved on understanding the mechanical, magnetic and other physical properties of nanostructures under an external magnetic field. Similar to investigations of effect of magnetic fields ${ }^{3}$ related to macroscopic materials, studies on the nanostructures are needed. The study of this subject may be necessary for future technological applications such as in nanoelectromechanical systems (NEMS), nanosensors, actuators, spintronics and nanocomposites. The study of nanostructures such as carbon nanotubes and graphene sheets under magnetic field has generated increasing interest among scientific researchers as evidenced from the literature. The interesting studies on the effect of magnetic fields on properties of nanostructures such as single-walled and multi-walled carbon nanotubes are reported recently. ${ }^{4,5}$ Studies such as on thermal, vibration, transport properties have also

*Author to whom correspondence should be addressed. been conducted on magnetic field and its influence on two-dimensional graphene. ${ }^{6-11}$ The emergence of magnetism in graphene and nanostructures has been found experimentally. ${ }^{12}$ Wang et al. ${ }^{13}$ recently illustrated the ferromagnetism of graphene-based materials at room temperature. Magnetic field at nanoscale is important and its influence can be tailored accordingly; consequently theoretical, computational and experimental research is this area is thus essential.

Axial vibrations of rods in a magnetic field are important in materials science and mechanical engineering. The effect of a magnetic field on Young's modulus has been demonstrated experimentally through axial vibrations of a macroscopic ferritic steel rod in a magnetic field. ${ }^{14}$ Studying the axial vibration of nickel rods using optical heterodyne interferometry, Chicharro et al. ${ }^{15}$ determined the change in elastic modulus with the applied magnetic field and the magnetoelastic coupling coefficient. The same group $^{16}$ further studied the magnetic field dependence of nickel materials and how damping affects the Young's modulus, through studying free and forced axial vibrations of a slender rod using optical heterodyne interferometry. 
They ${ }^{17}$ further used the same technique to investigate the relationship between Young's modulus and internal stresses in nickel.

Experimental work ${ }^{18}$ and atomistic simulations ${ }^{19,20}$ have shown a significant 'size-effect' in the mechanical and physical properties of structures when the dimensions become 'small.' Here, by 'small' we mean on the order of nanoscale. Size-effects are related to the noncontinuum nature of material interactions on a molecular scale. Molecular dynamics (MD) simulations ${ }^{21-24}$ are computationally expensive but classical continuum models are questionable in the analysis of 'smaller' structures. Thus size-dependent continuum theories are gaining importance. ${ }^{25}$ These theories bring in the sizeeffects or scale-effects within the formulation by amending traditional classical continuum mechanics. However these size dependent theories though appropriate for microstructures cannot be totally applied to nanostructures. One widely used size-dependant theory, the nonlocal elasticity theory pioneered by Eringen ${ }^{26}$ is widely used for nanoscale structures. Nonlocal elasticity accounts for the small-scale effects arising at the nanoscale level. In nonlocal elasticity theory, the small-scale effects are captured by assuming that the stress at a point is a function of the strains at all points in the domain. ${ }^{26}$ This differs from classical elasticity. Nonlocal elastic theory considers long-range inter-atomic interaction and yields results dependent on the size of a body. The application of nonlocal elasticity in the mechanical analysis of nanostructures is widely described in the literature. ${ }^{27-43}$ The reliability of the nonlocal approaches has also been validated by molecular dynamics simulations. ${ }^{44}$

Using classical beam and shell theories, and updated nonlocal elasticity theories, studies have been conducted on the dynamics of carbon nanotubes subjected to external longitudinal and transverse magnetic fields. Li et el. ${ }^{5}$ presented the effect of transverse magnetic fields on dynamic characteristics of multi-walled carbon nanotubes using continuum theory. Wang et al. ${ }^{45}$ considered the effect of longitudinal magnetic field on wave propagation in carbon nanotubes (CNTs) embedded in an elastic matrix based on continuum medium theories. Narendar et al. ${ }^{46}$ used nonlocal elasticity theory and Euler-Bernoulli beam theory to study wave propagation in single-walled carbon nanotubes in a longitudinal magnetic field. Recently Murmu et al. ${ }^{47}$ carried out free vibration analysis of double-walled carbon nanotubes subjected to a longitudinal magnetic field. Coupled scale effects and the influence of a longitudinal magnetic field on the free transverse vibration of nanotube systems were described in detail.

According to the literature, limited work is done on nonlocal elasticity and the influence of magnetic field on nanostructures. The study is in its infancy stage. Though work has been done on influence of longitudinal field on nonlocal nanostructures, the effect of transverse magnetic field on nanostructure such as nanorod (with and without elastic medium) is scarce.

Therefore in this paper the effects of an external transverse magnetic field on the axial vibration of a nanorod such as a carbon nanotube with and without the elastic medium are studied. The theoretically study of this topic is reported in the literature but has not been addressed exhaustively. Nonlocal elasticity is used to address the small scale-effects. The nanorods are considered magnetically sensitive. Using nonlocal rod theory, governing equations for nonlocal axial vibration are derived, considering the Lorentz magnetic forces induced by a transverse magnetic field through Maxwell equations. The nonlocal natural frequencies of the nanorod under a transverse magnetic field are obtained analytically by solving the governing equation. Clamped-clamped and clamped-free boundary conditions are considered. The combined effects of the magnetic field and the nonlocal treatment of the problem are illustrated and discussed.

\section{MAXWELL'S RELATIONS FOR CONDUCTING NANOSTRUCTURES}

We denote $J$ as current density, $h$ as distributing vector of the magnetic field, $e$ as strength vectors of the electric field, and $U$ the vector of displacements. The Maxwell relations for conducting nanostructure according to Refs. $[45,46,48]$ can be retrieved as

$$
\begin{gathered}
J=\nabla \times h \\
\nabla \times e=-\eta \frac{\partial h}{\partial t} \\
\nabla \cdot h=0 \\
e=-\eta\left(\frac{\partial U}{\partial t} \times H\right)
\end{gathered}
$$

The distributing vector of the magnetic field can be represented as

$$
h=\nabla \times(U \times H)
$$

where $\eta$ is the magnetic field permeability, $\nabla$ is the Hamiltonian operator, $(i, j, k)$ are the unit vectors and $H$ is the magnetic field vector.

The Lorentz forces in terms of the displacement vector are given as ${ }^{45,46}$

$$
f_{L}=f_{L x} \mathbf{i}+f_{L y} \mathbf{j}+f_{L z} \mathbf{k}=\eta(J \times H)
$$

The electric force effect on the Lorentz force is neglected here.

We assume only a transverse magnetic field as $H=$ $H\left(0, H_{y}, H_{z}\right)$. Thus the effective Lorentz resultant force on the magnetically sensitive nanorod of cross section $A$ is considered as

$$
f=\int_{A} \eta f_{L x} d A=\eta A\left(H_{y}^{2}+H_{z}^{2}\right)
$$




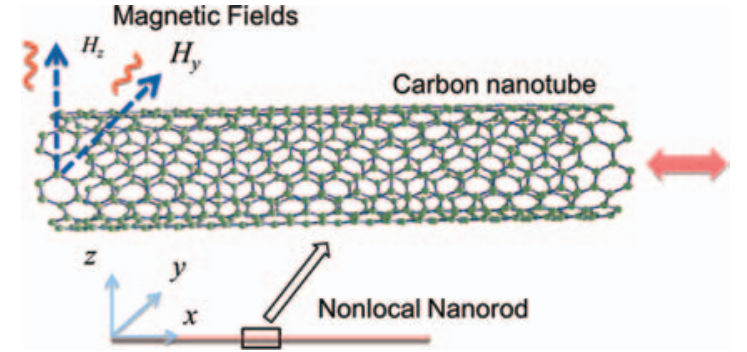

Fig. 1. Nonlocal nanorod such as carbon nanotube subjected to transverse magnetic field.

Terms $H_{y}$ and $H_{z}$ are the magnetic field strengths in the $y$ and $z$ directions (Fig. 1).

\section{NONLOCAL ROD UNDER TRANSVERSE MAGNETIC FIELD}

The nonlocal rod theory is based on a nonlocal elasticity approach. According to the theory the stress-strain relation is given as $^{26,28}$

$$
\left(1-\alpha^{2} \nabla_{L}^{2}\right) \sigma_{i j}(\mathbf{x})=\sigma_{i j}^{c}(\mathbf{x})=C_{i j k l} \varepsilon_{k l}(\mathbf{x})
$$

where $\alpha$ is the dimensional nonlocal parameter (of the order one nanometre) expressed as $\alpha=e_{0} a$, with $e_{0}$ a constant appropriate to each material and $a$ an internal characteristic length such as lattice parameter, inter-atomic distance or sample size. $\nabla_{L}$ is the Laplacian parameter.

Using Eq. (8), the nonlocal axial force $N$ is given as

$$
N-\left(e_{0} a\right)^{2} \frac{\partial^{2} N}{\partial x^{2}}=E A \frac{\partial u}{\partial x}
$$

where $u$ and $A$ are the axial displacement and the cross sectional area of the nanorod. $E$ denotes the sizeindependent Young's modulus of the nanorod. However it should be noted that at small scale, Young's modulus is size-dependent.

Using Eqs. (8) and (9), the governing equation of the nonlocal rod can be derived as ${ }^{28}$

$$
\begin{aligned}
& E A \\
& \quad+\left(f-\left(e_{0} a\right)^{2} \frac{\partial^{2} u(x, t)}{\partial x^{2}}-m \frac{\partial^{2} u(x, t)}{\partial t^{2}}+m\left(e_{0} a\right)^{2} \frac{\partial^{4} u(x, t)}{\partial x^{2} \partial t^{2}}\right. \\
& +(10
\end{aligned}
$$

We assume the effect of the transverse magnetic field on the magnetically sensitive nanorod is the experiencing of a Lorentz force. Utilizing Eqs. (8) and (9), the governing equation of a nonlocal rod subjected to transverse magnetic field is given as

$$
\begin{gathered}
E A \frac{\partial^{2} u(x, t)}{\partial x^{2}}-m \frac{\partial^{2} u(x, t)}{\partial t^{2}}+m\left(e_{0} a\right)^{2} \frac{\partial^{4} u(x, t)}{\partial x^{2} \partial t^{2}} \\
+\eta A\left(H_{y}^{2}+H_{z}^{2}\right)\left(\frac{\partial^{2} u}{\partial x^{2}}-\left(e_{0} a\right)^{2} \frac{\partial^{4} u}{\partial x^{4}}\right)=0
\end{gathered}
$$

\section{ANALYTICAL SOLUTIONS OF VIBRATING NANORODS}

Here we provide the analytical solution of the axial frequency of the nonlocal rod. Consider the solution of Eq. (11), which is of the form

$$
u(x, t)=U(x) \sin (\omega t)
$$

where $\omega$ is the circular frequency. Substituting Eq. (12) in Eq. (11) we obtain

$$
\begin{aligned}
& E A \frac{d^{2} U}{d x^{2}}+m \omega^{2} U-m\left(e_{0} a\right)^{2} \omega^{2} \frac{d^{2} U}{d x^{2}} \\
& +\eta A\left(H_{y}^{2}+H_{z}^{2}\right)\left(\frac{d^{2} U}{d x^{2}}-\left(e_{0} a\right)^{2} \frac{d^{4} U}{d x^{4}}\right)=0
\end{aligned}
$$

The above equation is expressed as

$$
P \frac{d^{4} U}{d x^{4}}+q \frac{d^{2} U}{d x^{2}}+r U=0
$$

This is a fourth-order differential equation. However, for the nonlocal rod we have two boundary condition equations. Thus for simplicity we neglect the fourth order term and obtain Eq. (14) as

$$
\frac{d^{2} U(x)}{d x^{2}}+\beta U(x)=0
$$

where

$$
\beta^{2}=\frac{m \omega^{2}}{E A+\eta A\left(H_{y}^{2}+H_{z}^{2}\right)-\left(e_{0} a\right)^{2} m \omega^{2}}
$$

The solution of Eq. (14) can be expressed in the form

$$
U(x)=A_{1} \cos \beta x+A_{2} \sin \beta x
$$

\section{Clamped-clamped Boundary Condition:}

The boundary conditions for the clamped-clamped case are given as $U(0)=0, U(L)=0$. Substituting in the boundary conditions, we get $A_{1}=0, A_{2} \sin \beta L=0$. Therefore the frequency equation is

$$
\sin \beta L=0
$$

and the roots are

$$
\beta L=r \pi, \quad r=1,2, \ldots
$$

Combining Eqs. (19) and (15) leads to

$$
\frac{m \omega^{2}}{E A+\eta A\left(H_{y}^{2}+H_{z}^{2}\right)-\left(e_{0} a\right)^{2} m \omega^{2}}=\left(\frac{r \pi}{L}\right)^{2}
$$

From Eq. (20) the natural frequency can be obtained explicitly as

$$
\omega=\sqrt{\frac{((\pi r) / L)^{2}\left[E A+\eta A\left(H_{y}^{2}+H_{z}^{2}\right)\right]}{m\left(1+\left(e_{0} a\right)^{2}((\pi r) / L)^{2}\right)}} \quad r=1,2, \ldots
$$


We re-write Eq. (21), by introducing the frequency parameter $\Omega(\psi, \alpha, r)=\sqrt{\left(m \omega^{2} L^{2}\right) /(E A)}$, as

$$
\Omega^{2}=\frac{(\pi r)^{2}\left[1+\psi\left(1+\delta^{2}\right)\right]}{\left[1+\alpha^{2}(\pi r)^{2}\right]}
$$

where the term $\psi=\left(\eta H_{y}^{2}\right) / E$ is a magnetic parameter; $H_{z}=\delta H_{y}$ and $\alpha=\left(e_{0} a\right) / L$ is the nonlocal parameter.

Clamped-Free Boundary Condition:

The boundary conditions of the clamped-free case are given as $U(0)=0, N(L)=0$, where $N$ is the axial force on the nanorod. The boundary condition at the free end (i.e., at $x=L$ ) is function of scale parameter $e_{0} a$ and magnetic field. Neglecting the magnetic field effects at the free end, the natural boundary conditions, can be reduced to $U^{\prime}(L)=0$. A variational basis of the model can be utilised in order to derive correct natural and essential boundary conditions.

Substituting the boundary conditions at the two ends we get $A_{1}=0, A_{2} \cos \beta L=0$.

Therefore the frequency equation is

$$
\cos \beta L=0
$$

The roots of this transcendental equation are

$$
\beta L=(2 r-1) \frac{\pi}{2}, \quad r=1,2, \ldots
$$

From Eq. (15) we get

$$
\frac{m \omega^{2}}{E A+\eta A\left(H_{y}^{2}+H_{z}^{2}\right)-\left(e_{0} a\right)^{2} m \omega^{2}}=\left[(2 r-1) \frac{\pi}{2 L}\right]^{2}
$$

Thus the natural frequency of the clamped-free nanorod is given by

$$
\begin{aligned}
& \omega=\sqrt{\frac{[(2 r-1) \pi / 2 L]^{2}\left(E A+\eta A\left(H_{y}^{2}+H_{z}^{2}\right)\right)}{m\left(1+\left(e_{0} a\right)^{2}[(2 r-1) \pi / 2 L]^{2}\right)}} \\
& r=1,2, \ldots
\end{aligned}
$$

Similarly Eq. (26) can be expressed as

$$
\Omega^{2}=\frac{[(2 r-1) \pi / 2]^{2}\left[1+\psi\left(1+\delta^{2}\right)\right]}{\left[1+\alpha^{2}((2 r-1) \pi / 2)^{2}\right]}
$$

\section{MAGNETIC FIELD AFFECTED NANOROD EMBEDDED IN ELASTIC MEDIUM}

Suppose the nanorod is embedded in an elastic medium and subjected to transverse magnetic field. The frequency for this case can be obtained as ${ }^{49}$

Clamped-clamped Case:

$$
\begin{aligned}
& \omega=\sqrt{\frac{((\pi r) / L)^{2}\left[E A+\eta A\left(H_{y}^{2}+H_{z}^{2}\right)\right]+k\left(1+\left(e_{0} a\right)^{2}((\pi r) / L)^{2}\right)}{m\left(1+\left(e_{0} a\right)^{2}((\pi r) / L)^{2}\right)}} \\
& r=1,2, \ldots .
\end{aligned}
$$

Similarly to above, we introduce the frequency parameter $\Omega(\psi, \alpha, r)=\sqrt{m \omega^{2} L^{2} / E A}$ and re-write Eq. (28) as

$$
\Omega^{2}=\frac{(\pi r)^{2}\left[1+\psi\left(1+\delta^{2}\right)\right]+K\left(1+\alpha^{2}(\pi r)^{2}\right)}{\left[1+\alpha^{2}(\pi r)^{2}\right]}
$$

Clamped-Free Case:

The natural frequency of the clamped-free nanorod is given by

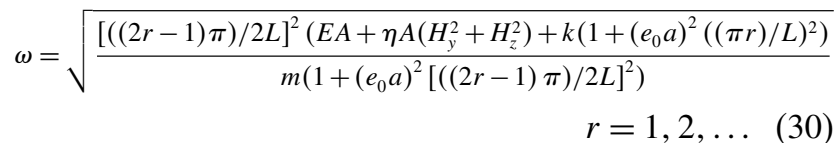

Similarly Eq. (30) can be expressed as

$\Omega^{2}=\frac{[((2 r-1) \pi) / 2]^{2}\left[1+\psi\left(1+\delta^{2}\right)\right]+K\left(1+\alpha^{2}(\pi r)^{2}\right)}{\left[1+\alpha^{2}(((2 r-1) \pi) / 2)^{2}\right]}$

When we ignore the magnetic effect and nonlocal effect we get the frequency of a classical axial rod. The consideration of other magnetic body force behaviour due to any inherent magnetic properties in a simple nanorod, charged rod, or ferromagnetic nanorod is a topic of open study. It should be noted that Eqs. (29) and (31) are approximate theoretical relations to represent a realistic nanorod (CNT) in a strong magnetic field where some conditions are assumed.

\section{MAGNETOELASTIC COUPLING}

The elastic moduli can be affected by an applied magnetic field. ${ }^{14-17}$ Experiments for macroscopic rods have been carried out to determine the relation for Young's modulus in a changing magnetic field. ${ }^{15}$ Consequences of magnetostriction in ferromagnetic rods are the magnetic field-dependence of the elastic modulus and coupling of the magnetic and elastic energies. A fundamental relation between these two effects can be given by

$$
\mu^{2}=1-\frac{E_{H}}{E_{W H}}
$$

where $\mu$ is the magnetoelastic coupling coefficient, $E_{H}$ and $E_{W H}$ are the Young's modulus that is magnetic field dependent and without magnetic field dependency. At a given frequency we have the relations.

Clamped-clamped case:

$$
\mu^{2}=\frac{\eta A\left(H_{y}^{2}+H_{z}^{2}\right)((\pi r) / L)^{2}}{m \omega^{2}\left(1+\left(e_{0} a\right)^{2}((\pi r) / L)^{2}\right)}
$$

Clamped-free case:

$$
\mu^{2}=\frac{\eta A\left(H_{y}^{2}+H_{z}^{2}\right)[((2 r-1) \pi) / 2 L]^{2}}{m \omega^{2}\left(1+\left(e_{0} a\right)^{2}[((2 r-1) \pi) / 2 L]^{2}\right)}
$$

It should be noted that the magnetoelastic coupling coefficient is a function of small scale effects also. The Eqs. (33) and (34) can provide the measure of sensitivity of Young's modulus to change to magnetic field. 


\section{RESULTS AND DISCUSSION}

\subsection{Nonlocal or Scale Parameter}

The applicability of nonlocal rod theory to nanotubes and nanorods is found in the literature. ${ }^{49}$ The validation of the nonlocal rod theory applied to carbon nanotubes has been carried out by comparing results with molecular dynamics simulations. ${ }^{44}$ The reliability of nonlocal rod theory depends on the value of nonlocal parameter or scale coefficient. Details of the values of nonlocal parameter reported by various researchers are discussed in Ref. [50]. Murmu and Adhikari ${ }^{44}$ have shown that a nonlocal parameter $e_{0} a=1.0$ matches well with molecular dynamics vibration analysis of carbon nanotubes. Thus nonlocal rod theory can be a reliable theory to predict the mechanical behaviour of nanorods when this optimised nonlocal parameter or scale coefficient is used. We have considered dimensionless nonlocal parameter in the range $0-1$ to cater to wide range of nonlocal effects. Here we discuss the effect of transverse magnetic field on the magnetically sensitive nanorod (CNT). The results are plotted in dimensionless parameters so as to address a wide range of values.
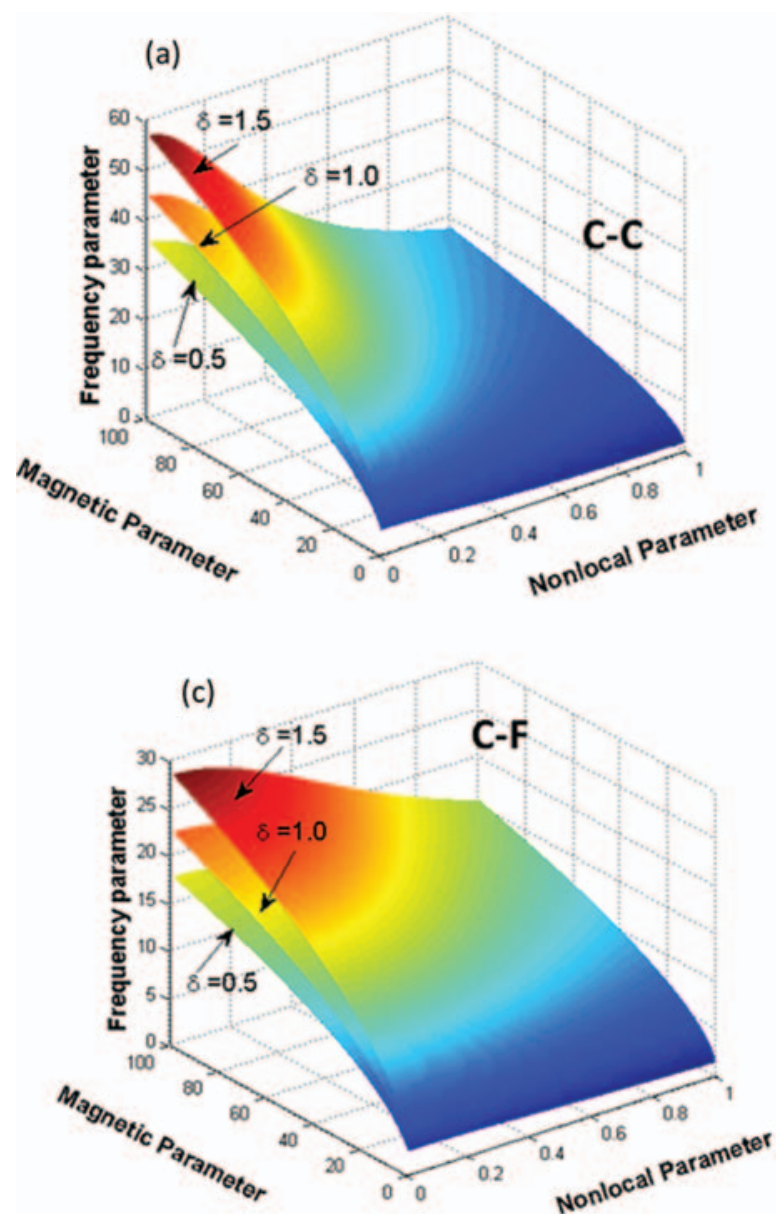

\subsection{Effect of Transverse Magnetic Field on Nanorod} Figures 2(a)-(d) show the plot of axial frequency parameter $\left(\Omega=\sqrt{\left.\left(m \omega^{2} L^{2}\right) /(E A)\right)}\right.$ of a nanorod (CNT), as a function of nonlocal parameter, $\alpha=\left(e_{0} a\right) / L$ and magnetic parameter $\psi=\left(\eta H_{y}^{2}\right) / E$. The nonlocal and magnetic parameters reflect the influence of small-scale effects and the transverse magnetic field on the nanorod respectively. Clamped-clamped and clamped free conditions are considered. As the nonlocal parameter increases, the frequency parameter decreases. Nonlocal elasticity predicts lower frequency compared to classical frequency. This is theoretically attributed to the softening of axial stiffness (Young's modulus) of the nanorod in the atomic scale regime. For second modes of vibration, the nonlocal effect is more pronounced and nonlinear at higher magnetic field strength. Further, Figure 2 shows that an increase in magnitude of the magnetic parameter (i.e., field strength) increases the frequency parameter. This can be attributed to the coupling effect of an axially vibrating nanorod and the transverse magnetic field. In a transverse magnetic field the axially vibrating nanorod may subjected to lower magnetic energy and it will hardly leave its equilibrium position if
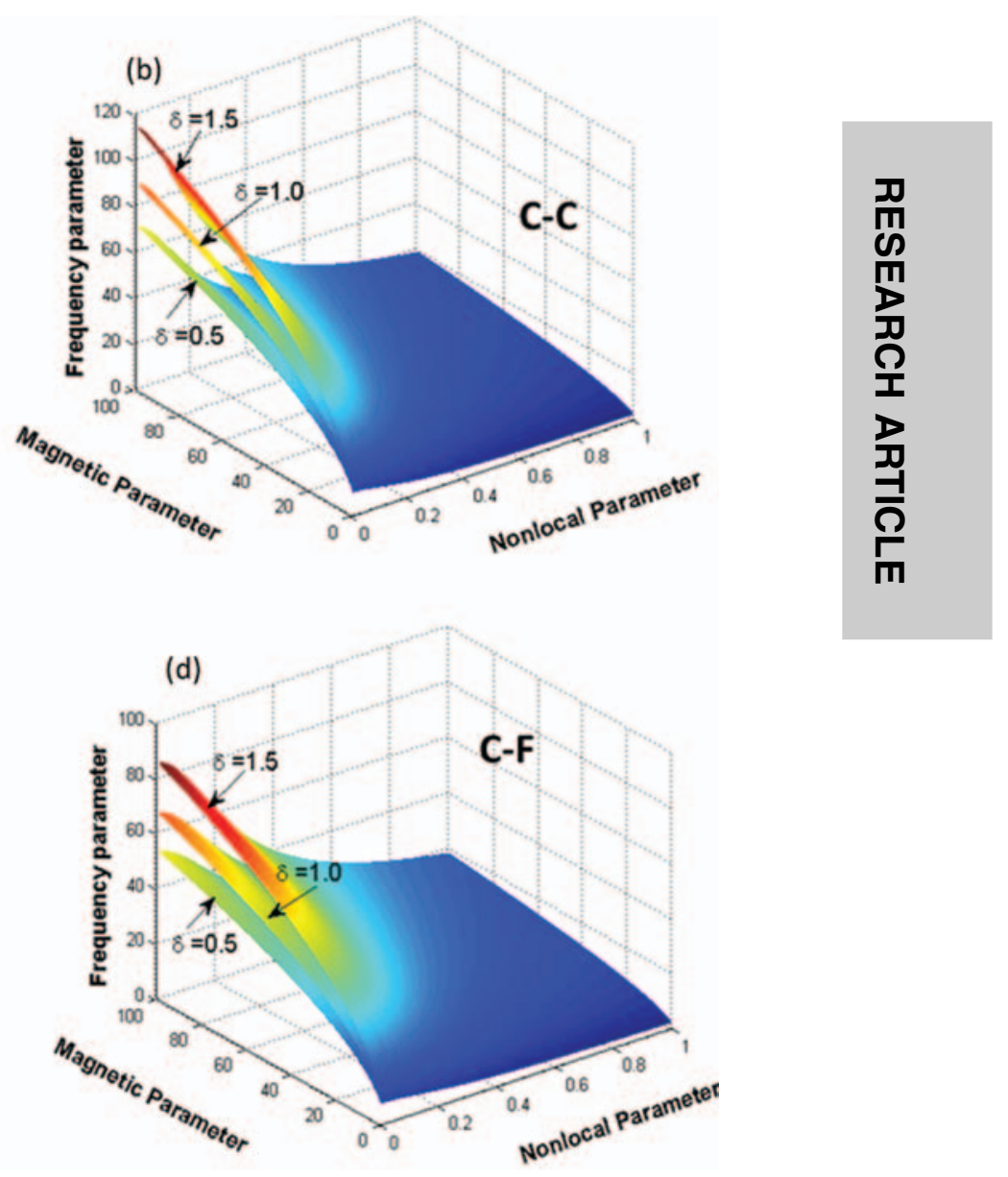

Fig. 2. Frequency parameter of an axially vibrating nanorod as a function of magnetic parameter and nonlocal parameter: (a) first frequency (clampedclamped), (b) second frequency (clamped-clamped), (c) first frequency (clamped-free) (d) second frequency (clamped-free). 

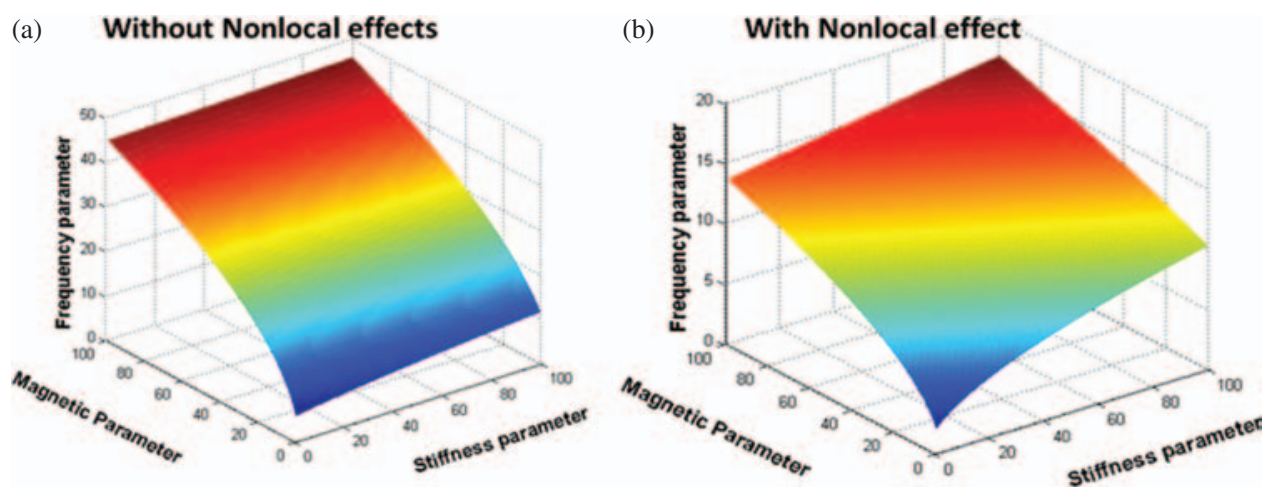

Fig. 3. Frequency parameter of an axially vibrating clamped-clamped nanorod surrounded by elastic medium is shown as a function of magnetic parameter and stiffness parameter: (a) without nonlocal effects (b) with nonlocal effects.

there is any slight disturbance, and no constrain of elastic deformation. And thus every point of nanorod will always tend to retreat to its position. Thus the frequency of axial vibration in a transverse field will increase. Further when some mechanism induces extra deformation either above or below the elastic deformation, the associated Young's modulus is different to normal. The magnetoelastic deformation produces an effect, making the nanorod more rigid. The directions of magnetic field and the vibration of nanorod, if in transverse directions, would result in the increase of the associated frequency. Figure 2 also shows that as the parameter $\delta\left(=H_{z} / H_{y}\right)$ increases the frequency parameter increases, and for higher values of $\delta$ the variation of frequency parameter with nonlocal parameter is more nonlinear in nature than for lower values of $\delta$.

\subsection{Embedded Nanorods in Transverse Magnetic Field}

Figures 3(a)-(b) shows the variation of frequency parameter with magnetic parameter and the stiffness parameter, $K$ of axially vibrating nanorod surrounded by an elastic medium (e.g., a polymer matrix). The frequency parameter increases with increasing stiffness of elastic medium. The influences of the elastic medium (e.g., polymer matrix) make the nanorod stiff and thus higher frequencies. The variation of frequency parameter with axial stiffness parameter of a nanorod in a magnetic field is more nonlinear with nonlocal effect than without nonlocal effects.

Molecular dynamics or experimental results for the present study are unavailable in literature. This represents a future scope of work. The present work deals with the continuum theory i.e., the nanotube is assumed to be continuous with specific organisation of matter. This paper thus considers a particular organization form of matter. Other forms are mentioned for example in the Refs. [51-53]. If we consider the subatomic regime in nonlocal mechanics, specifically the behaviour due to quantum effects, ${ }^{52,54}$ further study and upgrading in nonlocal mechanics is required. The present theoretical study of axial vibrations of nanorod enclosed in magnetic field could be important in material science and mechanical engineering. The material properties of nanostructures (e.g., CNTs) such as Young's modulus vary quite widely as found in the literature. The present theoretical study could aid in future experimental study of axial vibrations of nanorods in a magnetic field, with and without damping and internal stresses to throw light on the variation of the material properties of nanorods when in the presence of magnetic fields.

\section{CONCLUSION}

As axial vibrations of rod in magnetic field could be in material science and mechanical engineering, this communication reports the theoretical study on the influence of a transverse magnetic field on the axial vibration of a nanorod using equivalent continuum nonlocal rod theory. Axial frequencies turned out to be lower using nonlocal elasticity theory compared to the classical elasticity theory. Proposed theoretical results show that the transverse magnetic field exerted on the nanorod weakens the nonlocal effect of atom-atom interactions by increasing the natural frequencies. The variation of frequency parameter with axial stiffness parameter of a nanorod in a magnetic field and elastic medium is more nonlinear with nonlocal effect than without nonlocal effects. However the magnitudes of the natural frequencies are dependent on the choice of nonlocal parameter. The present work is useful for experimental study of the magnetic field dependent Young's modulus of magnetically sensitive nanorods with and without elastic medium.

\section{References}

1. T. Ono, Y. Fujimoto, and S. Tsukamoto, Quantum Matter 1, 4 (2012).

2. B. Tüzün and C. Erkoç, Quantum Matter 1, 136 (2012).

3. N. Paitya, S. Bhattacharya, D. De, and K. P. Ghatak, Quantum Matter 1, 63 (2012).

4. S. Bellucci, J. Gonzalez, F. Guinea, P. Onorato, and E. Perfetto, Journal of Physics-Condensed Matter 19 (2007).

5. S. Li, H. Xie, and X. Wang, Bulletin of Materials Science 34, 45 (2011) 
6. F. Peng, Physica Status Solidi B-Basic Solid State Physics 248, 1388 (2011).

7. C. Faugeras, P. Kossacki, D. Basko, M. Amado, M. Sprinkle, C. Berger, W. de Heer, and M. Potemski, Physical Review B 81 (2010).

8. Z. Fu, Z. Wang, S. Li, and P. Zhang, Chinese Physics B 20 (2011)

9. S. Kumar, M. Jalil, S. Tan, and G. Liang, Journal of PhysicsCondensed Matter 22 (2010).

10. F. Lopez-Urias, J. Rodriguez-Manzo, E. Munoz-Sandoval, M. Terrones, and H. Terrones, Optical Materials 29, 110 (2006)

11. K. Shizuya, Physical Review B 75 (2007).

12. O. Yazyev, Reports on Progress in Physics 73 (2010).

13. Y. Wang, Y. Huang, Y. Song, X. Zhang, Y. Ma, J. Liang, and Y. Chen, Nano Lett. 9, 220 (2009).

14. B. Kardashev, K. Van Ouytsel, and R. De Batist, Journal of Alloys and Compounds 310, 169 (2000).

15. J. Chicharro, A. Bayon, and F. Salazar, Journal of Magnetism and Magnetic Materials 202, 465 (1999).

16. J. Chicharro, A. Bayon, and F. Salazar, Journal of Magnetism and Magnetic Materials 268, 348 (2004).

17. J. Chicharro, A. Bayon, and F. Salazar, Journal of Magnetism and Magnetic Materials 297, 44 (2006).

18. C. Kiang, M. Endo, P. Ajayan, G. Dresselhaus, and M. Dresselhaus, Phys. Rev. Lett. 81, 1869 (1998).

19. C. Tang, L. Meng, L. Sun, K. Zhang, and J. Zhong, J. Appl. Phys. 104 (2008).

20. C. Tang, W. Guo, and C. Chen, Physical Review B 79 (2009).

21. K. Bi, Y. Chen, J. Yang, Y. Wang, and M. Chen, Phys. Lett. A 350, 150 (2006)

22. A. Brodka, J. Koloczek, and A. Burian, J. Nanosci. Nanotechnol. 7, 1505 (2007)

23. T. Han, P. He, J. Wang, and A. Wu, New Carbon Materials 25, 261 (2010).

24. R. Li, Y. Hu, H. Wang, and Y. Zhang, Acta Physica Sinica 55, 5455 (2006).

25. B. Akgoz and O. Civalek, International Journal of Engineering Science 49, 1268 (2011).

26. A. Eringen, J. Appl. Phys. 54, 4703 (1983).

27. R. Ansari, H. Rouhi, and S. Sahmani, International Journal of Mechanical Sciences 53, 786 (2011).

28. M. Aydogdu, Physica E-Low-Dimensional Systems and Nanostructures 41, 861 (2009).
29. M. Aydogdu and S. Filiz, Physica E-Low-Dimensional Systems and Nanostructures 43, 1229 (2011).

30. S. Filiz and M. Aydogdu, Computational Materials Science 49, 619 (2010).

31. H. Heireche, A. Tounsi, A. Benzair, M. Maachou, and E. Bedia, Physica E-Low-Dimensional Systems and Nanostructures 40, 2791 (2008).

32. J. Hsu, H. Lee, and W. Chang, Current Applied Physics 11, 1384 (2011).

33. M. Simsek, International Journal of Engineering Science 48, 1721 (2010).

34. Q. Wang, J. Appl. Phys. 98 (2005).

35. Q. Wang and V. Varadan, Smart Mater. Struct. 15, 659 (2006).

36. Q. Wang and K. Liew, Phys. Lett. A 363, 236 (2007).

37. Q. Wang and C. Wang, Nanotechnology 18 (2007).

38. R. Aghababaei and J. Reddy, Journal of Sound and Vibration 326, 277 (2009).

39. R. Ansari, S. Sahmani, and B. Arash, Phys. Lett. A 375, 53 (2010).

40. R. Ansari and H. Ramezannezhad, Physica E-Low-Dimensional Systems and Nanostructures 43, 1171 (2011).

41. R. Ansari, B. Arash, and H. Rouhi, Compos. Struct. 93, 2419 (2011).

42. S. Narendar and S. Gopalakrishnan, J. Comput. Theor. Nanosci. 7, 2349 (2010).

43. W. Chang and H. Lee, J. Comput. Theor. Nanosci. 7, 2367 (2010).

44. T. Murmu and S. Adhikari, Mechanics Research Communications 38, 62 (2011).

45. H. Wang, K. Dong, F. Men, Y. Yan, and X. Wang, Applied Mathematical Modelling 34, 878 (2010).

46. S. Narendar, S. Gupta, and S. Gopalakrishnan, Applied Mathematical Modelling Corrected Proof (2012).

47. T. Murmu, M. McCarthy, and S. Adhikari, Journal of Sound and Vibration 331, 5069 (2012)

48. J. Kraus, Electromagnetics, McGrawHill, Inc., USA (1984).

49. M. Aydogdu, Mechanics Research Communications 43, 34 (2012).

50. S. Narendar and S. Gopalakrishnan, Journal of Applied MechanicsTransactions of the Asme 78 (2011).

51. Q. Zhao, Reviews in Theoretical Science 1, 83 (2013).

52. A. Herman, Reviews in Theoretical Science 1, 3 (2013).

53. E. L. Pankratov and E. A. Bulaeva, Reviews in Theoretical Science 1, 58 (2013).

54. A. Khrennikov, Reviews in Theoretical Science 1, 34 (2013).

Received: 26 March 2013. Accepted: 20 April 2013. 